Pacific Journal of Mathematics

ON SOLUTIONS IN THE REGRESSIVE IDOLS 


\title{
ON SOLUTIONS IN THE REGRESSIVE ISOLS
}

\author{
JOSEPH BARBACK
}

Let $f(x)$ be a recursive function and let $D_{f}(X)$ denote the Nerode canonical extension of $f$ to the isols. Let $A$ and $Y$ be particular isols such that $D_{f}(A)=Y$. The main results in the paper deal with the following problem: if one of the isols $A$ and $Y$ is regressive, what regressive property if any will the other isol have. It is shown that if $A$ is a regressive isol then $Y$ will be also. Also, it is possible for $Y$ to be a regressive isol while $A$ is not. In this event there exist regressive isols $B$ with $D_{f}(B)=Y$ and $B \leqq_{A} A$. Extensions of these results for recursive functions of more than one variable are discussed in the last section of the paper.

1. Introduction. We will assume that the reader is familiar with the primary definitions and results of the papers listed as references. We will cite some particular definitions and results that have a special role in the paper. $E$ will denote the set of nonnegative integers, $A$ the collection of isols, $\Lambda^{*}$ the collection of isolic integers, and $\Lambda_{R}$ the collection of regressive isols. If $f$ is a partial function from a subset of $E$ into $E$ then $\delta f$ will denote its domain. If $f: E^{n} \rightarrow$ $E$ is a recursive function then $D_{f}$ will denote the canonical extension of $f$ to the isols. Two sets $\alpha$ and $\beta$ will be separated, written $\alpha \mid \beta$, if there exist disjoint r.e. supersets of $\alpha$ and $\beta . j(x, y)$ will denote the familiar recursive pairing function defined by,

$$
j(x, y)=x+1 / 2(x+y)(x+y+1),
$$

and $k$ and $l$ the associated functions with the property $j(k(x), l(x))=$ $x$. $\left[\rho_{x}\right]$ will be the canonical enumeration for the collection of all finite subsets of $E$, [6]. Associated with this enumeration is the recursive function $r(x)$ having the property $r(x)=$ card $\rho_{x}$. We will use a $\sum$ to stand for union among sets (and also $a+$ for a union of two sets).

2. Recursive functions of one variable. Let $f: E \rightarrow E$ be a recursive function. If $f$ is a combinatorial function then its extension $D_{f}$ will map $\Lambda$ into $\Lambda$, and if $f$ is an increasing function then $D_{f}$ will map $\Lambda_{R}$ into $\Lambda_{R}$. Each combinatorial function of one variable will be increasing, but not conversely. The condition needed for $D_{f}$ to map $\Lambda_{R}$ into $\Lambda_{R}$ is that $f$ be an eventually increasing function, [1].

THEOREM 1. Let $f: E \rightarrow E$ be a recursive function and $A$ and $Y$ be 
isols such that $D_{f}(A)=Y$. If $A$ is a regressive isol then $Y$ will be regressive also.

Proof. Assume $A$ is a regressive isol. Let

$$
\begin{aligned}
g(0) & =0, \\
g(n+1) & =f(n)+g(n) .
\end{aligned}
$$

Then $g$ will be an increasing and recursive function. Hence its canonical extension $D_{g}$ will map $\Lambda_{R}$ into $\Lambda_{R}$. Since

$$
g(n+1)=f(n)+g(n),
$$

it follows from the Nerode metatheorem for such identities (combining [12, Theorem 10.1] and the representation of the canonical extension of a recursive function [11, 4]), that

$$
D_{g}(A+1)=D_{f}(A)+D_{g}(A) .
$$

Because $A$ is a regressive isol and $g$ is increasing and recursive, each of the isols $A+1, D_{g}(A+1)$ and $D_{g}(A)$ will also be regressive. In addition, $Y=D_{f}(A)$ is an isol and from (1) it then follows

$$
Y \leqq D_{g}(A+1) \text { and } D_{g}(A+1) \in \Lambda_{R} \text {. }
$$

In view of a result due to Dekker [4, P8 (a)], (2) implies that $Y$ will be a regressive isol.

REMARK. If $f$ is a recursive function of one variable then although its canonical extension may not map every isol onto an isol, its value may be an isol for some. In addition, it may also occur that the value of $D_{f}(A)$ will be a regressive isol for an isol $A$ which is nonregressive. An example of such a recursive function will be given in the following section. We want to show next that if this possibility does occur, then there will be a regressive isol $B$ such that $D_{f}(B)=$ $D_{f}(A)$. The following lemma essentially gives this result, once the connection is made between the canonical extensions of recursive functions and recursive combinatorial functions.

Lemma. Let $f, g: E \rightarrow E$ be recursive combinatorial functions and $A$ and $Y$ be isols which satisfy the identity,

$$
D_{f}(A)=Y+D_{g}(A) .
$$

If $Y$ is a regressive isol, then there will also exist a regressive isol $B$ with,

$$
D_{f}(B)=Y+D_{g}(B)
$$


Proof. Assume that $Y$ is a regressive isol, and consider separately the following three cases.

Case 1. $A$ is finite. Then $A$ will be regressive and we may set $B=A$.

Case 2. $A$ is infinite and $Y$ is finite. Let $Y=p \in E$. Set

$$
h(x)=p+g(x) \text {, for } x \in E .
$$

Then $h$ will be a recursive combinatorial function, since the function $g$ is recursive and combinatorial. By a theorem of Myhill and Nerode [11, Theorem 7], we also obtain,

$$
D_{h}(A)=Y+D_{g}(A) .
$$

Combining (1) and (3) implies

$$
D_{f}(A)=D_{h}(A),
$$

and since $A$ is an infinite isol, it follows from (4) and a theorem due to Myhill [8], that there will be infinitely many numbers $n$ that satisfy

$$
f(n)=h(n) \text {. }
$$

Let $m$ be the smallest number that satisfies (5), and let $B=m$. Then $B$ will be a regressive solution to (2), since

$$
\begin{aligned}
D_{f}(m) & =f(m) \\
& =h(m) \\
& =D_{h}(m) \\
& =p+D_{g}(m) \\
& =Y+D_{g}(m) .
\end{aligned}
$$

Case 3. Both $A$ and $Y$ are infinite isols. Let $\varphi_{f}$ and $\varphi_{g}$ be the normal combinatorial operators, and let $\left[c_{i}\right]$ and $\left[d_{i}\right]$ be the sequences of combinatorial coefficients that are associated with the functions $f$ and $g$ respectively. Let $\alpha \in A$ and $\eta \in Y$. Then $\alpha$ and $\eta$ will each be infinite and isolated sets, and also $\eta$ will be regressive. We will assume that

$$
\eta \mid \alpha \text { and } \eta \mid \varphi_{g}(\alpha)
$$

for otherwise an easy modification may be made in the proof. Based on their respective definitions, each of the functions $c_{i}$ and $d_{i}$ will be recursive, and also 


$$
\begin{aligned}
& \varphi_{f}(\alpha)=\left(j(x, y) \mid \rho_{x} \subseteq \alpha \text { and } y<c_{r(x)}\right), \\
& \varphi_{g}(\alpha)=\left(j(x, y) \mid \rho_{x} \leqq \alpha \text { and } y<d_{r(x)}\right) .
\end{aligned}
$$

From (1) and (6) it follows also,

$$
\varphi_{f}(\alpha) \cong \eta+\varphi_{g}(\alpha) .
$$

Let $p$ be a partial recursive function that establishes (7), i.e., $p$ will be defined on $\varphi_{f}(\alpha)$, will be one-to-one, and will map

$$
p: \varphi_{f}(\alpha) \rightarrow \eta+\varphi_{g}(\alpha),
$$

one-to-one and onto.

Let $y_{x}$ be a regressive function that ranges over the set $\eta$.

Our first aim is to define two particular sequences of subsets of $\alpha$ and of $\eta$ respectively, whose corresponding terms will share the property appearing in (8). With each number $n$ we will associate two sets $\alpha_{n}$ a subset of $\alpha$, and $\eta_{n}$ a subset of $\eta$. These sets are meant to be the collections of those members of $\alpha$ and $\eta$ respectively, that we can effectively find if we start with the value of $y_{n}$ and use only the regressive property of the function $y_{x}$, the separability property in (6), and the recursive and partial recursive properties that appear in (8). Note that the inverse function $p^{-1}$ of $p$ will be well-defined and partial recursive. The particular definition for these sets is as follows; for $n \in E$, the members of $\alpha_{n}$ and $\eta_{n}$ are determined by repeated applications of the six rules below,

(i) $y_{n} \in \eta_{n}$,

(ii) if $y_{k} \in \eta_{n}$ then $\left(y_{0}, \cdots, y_{k}\right) \subseteq \eta_{n}$,

(iii) if $y_{k} \in \eta_{n}$ and $p^{-1}\left(y_{k}\right)=j(x, u)$, then $\rho_{x} \subseteq \alpha_{n}$,

(iv) if $a_{1}, \cdots, a_{k} \in \alpha_{n}, \rho_{x}=\left(a_{1}, \cdots, a_{k}\right), y<c_{k}, p j(x, y) \in \eta$ and $p j(x$, $y)=y_{m}$, then $y_{m} \in \eta_{n}$,

(v) if $\alpha_{1}, \cdots, a_{k} \in \alpha_{n}, \rho_{x}=\left(a_{1}, \cdots, a_{k}\right), y<c_{k}$ and $p j(x, y)=j(u$, $v)$, then $\rho_{u} \subseteq \alpha_{n}$,

(vi) if $a_{1}, \cdots, a_{k} \in \alpha_{n}, \rho_{x}=\left(a_{1}, \cdots, a_{k}\right), y<d_{k}$ and $p^{-1} j(x, y)=j(u$, $v)$, then $\rho_{u} \subseteq \alpha_{n}$.

Note that each of the sets $\eta_{n}$ will be non-empty, in view of (i). It may occur that some of the sets $\alpha_{n}$ are empty, however this will be true for at most only finitely many of the $\alpha_{n}$. It is easy to see upon a moments reflection that from the value of the number $y_{n}$ one can effectively enumerate all of the members in each of the sets $\alpha_{n}$ and $\eta_{n}$. It follows that each of the sets $\alpha_{n}$ and $\eta_{n}$ (for any number $n$ ) will be r.e. subsets of $\alpha$ and $\eta$ respectively. Since $\alpha$ and $\eta$ are each isolated sets, we see that each of the sets $\alpha_{n}$ and $\eta_{n}$ will be finite. It will be useful to list some of these properties and also some that 
can be arrived at in an easy manner from the six rules above.

$$
\begin{gathered}
(\forall n)\left[\eta_{n} \neq \varnothing\right] \text { and }(\forall n)(\exists k)\left[\alpha_{n+k} \neq \varnothing\right] . \\
(\forall n)(\exists t)\left[t \geqq n \text { and } \eta_{n}=\left(y_{0}, \cdots, y_{t}\right)\right] . \\
\alpha_{0} \subseteq \alpha_{1} \subseteq \alpha_{2} \subseteq \cdots \text { and } \sum_{0}^{\infty} \alpha_{n} \subseteq \alpha . \\
\eta_{0} \subseteq \eta_{1} \subseteq \eta_{2} \subseteq \cdots \text { and } \sum_{0}^{\infty} \eta_{n}=\eta
\end{gathered}
$$

In addition, note that the six rules (i) - (vi) have been so defined so have the following property; if one would simply know only the value of $y_{n}$, then the totality of those members of $\alpha$ and $\eta$ that could be found by using only the recursive and regressive features present in (8) would be the two sets $\alpha_{n}$ and $\eta_{n}$ respectively. It follows from this property that, for $n \in E$

$$
p: \varphi_{f}\left(\alpha_{n}\right) \rightarrow \eta_{n}+\varphi_{g}\left(\alpha_{n}\right), \text { one-to-one and onto. }
$$

For each number $n \in E$, let the

$$
\text { torre number of } \eta_{n}=\text { the largest number } t \text { with } \eta_{t}=\eta_{n} \text {. }
$$

In view of (i) and the fact that each of the sets $\eta_{n}$ is finite, it follows that there will be infinitely many torre numbers. In addition it is easy to see that if $t$ is the torre number of $\eta_{n}$, then $t \geqq n$ and $\eta_{t}=\eta_{n}=\left(y_{0}, \cdots, y_{t}\right)$. Let $t_{x}$ denote the strictly increasing function that ranges over the set of all torre numbers. Then

$$
\begin{gathered}
\eta_{t_{x}}=\left(y_{0}, \cdots, y_{t_{x}}\right), \\
\eta_{t_{0}} \subseteq \eta_{t_{1}} \leqq \eta_{t_{2}} \subseteq \cdots, \\
t_{x}<k \leqq t_{x+1} \Longrightarrow \eta_{k}=\eta_{t_{x+1}}, \text { and } \\
\eta=\sum_{0}^{\infty} \eta_{t_{n}} .
\end{gathered}
$$

In addition, by combining the remark prior to (13) with (16) and the fact that $y_{n}$ is a regressive function, we can also see that $y_{t_{x}}$ will be a regressive function (of $x$ ). This turns out to be a very useful property. Another fact that is important to note here is property $A$ given below; it follows from (13), (16), the definitions of $\eta_{n}$ and its torre number, and the regressive property of $y_{t_{x}}$.

Property $A$. If we are given the value of $y_{k}$ then we can effectively determine whether $k \leqq t_{0}$ or there is a number $x$ such that $t_{x}<$ $k \leqq t_{x+{ }_{1}}$. In the former event we could also find the value of $y_{t_{0}}$, 
and in the latter event both of the numbers $y_{t_{x}}$ and $y_{t_{x+1}}$ could be found.

Combining (11), (13) and (15) gives,

$$
\begin{aligned}
& \alpha_{t_{0}} \subseteq \alpha_{t_{1}} \subseteq \alpha_{t_{2}} \subseteq \cdots, \text { and } \\
& p: \varphi_{f}\left(\alpha_{t_{x}}\right) \longrightarrow \eta_{t_{x}}+\varphi_{g}\left(\alpha_{t_{x}}\right),
\end{aligned}
$$

one-to-one and onto, for each number $x$. Since $\varphi_{f}$ and $\varphi_{g}$ are combinatorial operaors, the inclusions appearing in (18) also imply that

$$
\varphi_{f}\left(\alpha_{t_{x}}\right) \subseteq \varphi_{f}\left(\alpha_{t_{x+1}}\right),
$$

and

$$
\varphi_{g}\left(\alpha_{t_{x}}\right) \subseteq \varphi_{g}\left(\alpha_{t_{x+1}}\right) \text {. }
$$

Therefore, in view of (15) and (19), we obtain for each number $x \in E$,

$$
\begin{gathered}
p:\left(\varphi_{f}\left(\alpha_{t_{x+1}}\right)-\varphi_{f}\left(\alpha_{t_{x}}\right)\right) \\
\longrightarrow\left(\eta_{t_{x+1}}-\eta_{t_{x}}\right)+\left(\varphi_{g}\left(\alpha_{t_{x+1}}\right)-\left(\varphi_{g}\left(\alpha_{t_{x}}\right)\right),\right.
\end{gathered}
$$

one-to-one and onto.

We now begin to design a regressive set $\beta$ whose recursive equivalence type will have the desired properties of the lemma. First with each number $y_{t_{x}}$ a particular finite set $\beta_{x}$ will be associated. Let the functions $w_{x}$ and $e_{x}$ be defined by

$$
\begin{aligned}
w_{x} & =\text { cardinality of } \alpha_{t_{x}}, \\
e_{0} & =w_{0}, \\
e_{n+1} & =w_{n+1}-w_{n} .
\end{aligned}
$$

Since $y_{t_{x}}$ is a regressive function and since from the value of $y_{t_{x}}$ we can determine the complete set $\alpha_{t_{x}}$ (refer to the remarks appearing before (13)), we see that from the value of $y_{t_{x}}$ alone, each of the numbers $w_{x}$ and $e_{x}$ can be computed. Hence each of the mappings $y_{t_{n}} \rightarrow w_{n}$ and $y_{t_{n}} \rightarrow e_{n}$ will have a partial recursive extension; in the notation of [4] these properties are denoted respectively by

$$
y_{t_{n}} \leqq w_{n} \text { and } y_{t_{n}} \leqq * e_{n} \text {. }
$$

We will assume here that $e_{0} \geqq 1$ (otherwise the proof would need to be slightly changed). Then, by (18), it will also follow that $e_{n} \geqq 1$ for each number $n$. For $n \in E$, let

$$
\delta_{n}=\left[j\left(y_{t_{n}}, r\right) \mid r=0,1, \cdots, e_{n}-1\right] .
$$

Then $\left[\delta_{n}\right]$ will be a sequence of mutually disjoint nonempty sets. From 
(21) and (22), we see that by knowing the value of $y_{t_{n}}$ we can effectively find all the members of the set $\delta_{n}$; this property will be denoted by writing

$$
y_{t_{n}} \leqq \delta_{n}
$$

For $n \in E$ set

$$
\beta_{n}=\delta_{0}+\delta_{1}+\cdots+\delta_{n} .
$$

Then, in view of (23) and the regressiveness of $y_{t_{n}}$, it is possible to effectively find all the elements of $\beta_{n}$ from the value of $y_{t_{n}}$. We will denote this property by

$$
y_{t_{n}} \leqq \beta_{n}
$$

In addition, note that

$$
\begin{gathered}
\beta_{0} \leqq \beta_{1} \leqq \beta_{2} \leqq \cdots, \text { and } \\
\text { card } \beta_{x}=\text { card } \alpha_{t_{x}} \text { for every } x \in E .
\end{gathered}
$$

Let

$$
\beta=\sum_{0}^{\infty} \beta_{n}=\sum_{0}^{\infty} \delta_{n}
$$

We will assume here that the sets $\eta$ and $\varphi_{g}(\beta)$ are separated (otherwise an easy change in the proof would be made), i.e.,

$$
\eta \mid \varphi_{g}(\beta) \text {. }
$$

Let $B=\operatorname{Req} \beta$. The remainder of the discussion now is toward showing that $B$ will satisfy the desired requirements of the lemma, i.e., that $B$ is a regressive isol and that $B$ satisfies (2). Observe that by (28),

$$
\eta+\varphi_{g}(\beta) \in Y+D_{g}(B) \text {. }
$$

Hence in order to complete the proof, it suffices to show that

$$
\beta \text { is a regressive and isolated set, and }
$$

$$
\varphi_{f}(\beta) \cong \eta+\varphi_{g}(\beta) \text {. }
$$

For (29): Note that $\beta$ will be an infinite set, since $e_{n} \geqq 1$ for each number $n$. Also, it is easy to see that if $\beta$ contains an infinite r.e. subset, then the set $\left(y_{t_{0}}, y_{t_{1}}, \cdots\right)$ would also then include an infinite r.e. subset. But then the set $\eta$ would contain an infinite r.e. subset, yet we know that this cannot be true since it is an isolated set. And therefore we may conclude that $\beta$ will be an isolated set. We know that the function $y_{t_{x}}$ is regressive. If we combine this fact with (23) and the definition of $\beta$, then it is easy to see that $\beta$ will 
be a regressive set, and in particular that a regressive enumeration of its members will be

$$
j\left(y_{t_{0}}, 0\right), \cdots, j\left(y_{t_{i}}, e_{0}-1\right), j\left(y_{t_{1}}, 0\right), \cdots, j\left(y_{t_{1}}, e_{1}-1\right), \cdots .
$$

For (30): Recall that

$$
\beta=\sum_{0}^{\infty} \beta_{n} \text { where } \beta_{n}=\delta_{0}+\cdots+\delta_{n} .
$$

Because $\varphi_{f}$ and $\varphi_{g}$ are combinatorial operators, it follows from (26) and (31) that,

$$
\begin{aligned}
& \varphi_{f}\left(\beta_{0}\right) \subseteq \varphi_{f}\left(\beta_{1}\right) \subseteq \cdots \text { and } \varphi_{f}(\beta)=\sum_{0}^{\infty} \varphi_{f}\left(\beta_{n}\right), \\
& \varphi_{g}\left(\beta_{0}\right) \subseteq \varphi_{g}\left(\beta_{1}\right) \subseteq \cdots \text { and } \varphi_{g}(\beta)=\sum_{0}^{\infty} \varphi_{g}\left(\beta_{n}\right),
\end{aligned}
$$

and also, in view of (19) and (27), that for $n \in E$,

$$
\operatorname{card} \varphi_{f}\left(\beta_{n}\right)=\operatorname{card} \eta_{t_{n}}+\operatorname{card} \varphi_{g}\left(\beta_{n}\right) \text {. }
$$

Combining (15), (32), (33) and (34) gives

$$
\begin{aligned}
\operatorname{card} \varphi_{f}\left(\beta_{0}\right) & =\operatorname{card} \eta_{t_{0}}+\operatorname{card} \varphi_{g}\left(\beta_{0}\right), \text { and } \\
\operatorname{card}\left(\varphi_{f}\left(\beta_{k+1}\right)\right. & \left.-\varphi_{f}\left(\beta_{k}\right)\right)=\operatorname{card}\left(\eta_{t_{k+1}}-\eta_{t_{k}}\right) \\
& +\operatorname{card}\left(\varphi_{g}\left(\beta_{k+1}\right)-\varphi_{g}\left(\beta_{k}\right)\right) \cdot
\end{aligned}
$$

Now we can define a partial function,

$$
q: \varphi_{f}(\beta) \longrightarrow \eta+\varphi_{g}(\beta),
$$

based on the previous two equations. Let

$$
\begin{gathered}
q: \varphi_{f}\left(\beta_{0}\right)-* \rightarrow \eta_{t_{0}}+\varphi_{g}\left(\beta_{0}\right), \\
q:\left(\varphi_{f}\left(\beta_{k+1}\right)-\varphi_{f}\left(\beta_{k}\right)\right)-* \rightarrow\left(\eta_{t_{k+1}}-\eta_{t_{k}}\right)+\left(\varphi_{g}\left(\beta_{k+1}\right)-\varphi_{g}\left(\beta_{k}\right)\right),
\end{gathered}
$$

where we write $-* \rightarrow$ to mean that the related mapping is to be order preserving. From (35) and (36) it follows that the mapping $q$ is well-defined, and from (12), (32) and (33) that $q$ will map $\varphi_{f}(\beta)$ onto $\eta+\varphi_{g}(\beta)$ in a one-to-one manner. To verify (30), it suffices to prove that $q$ will have a one-to-one partial recursive extension. Because the sets $\varphi_{f}(\beta)$ and $\eta+\varphi_{g}(\beta)$ are isolated, it follows from a theorem due to Dekker [4, Proposition $9(b)$ ], that $q$ will have a oneto-one partial recursive extension, if both $q$ and $q^{-1}$ have partial recursive extensions. It suffices therefore to verify this latter property, and this will be our approach here. We will consider first the mapping $q$. 
Let $w \in \varphi_{f}(\beta)$. We now describe a procedure whereby, with the possible exception of finitely many such $w$, one can effectively compute the value of $q(w)$. From $w$ first find the particular numbers $x$ and $u$ with

$$
w=j(x, u), \rho_{x} \cong \beta \text { and } u<c_{r(x)} .
$$

Note that if $\rho_{x}$ is nonempty then each of its members can also be found. Moreover, since $\varphi_{f}$ is a normal combinatorial operator, it follows that for all but possibly finitely many $w \in \varphi_{f}(\beta)$ the corresponding finite set $\rho_{x}$ appearing in (37) will be nonempty. From now on let us assume that $\rho_{x}$ is nonempty. Members of $\rho_{x}$ will be of the form $j\left(y_{t_{k}}, v\right)$, and for each such member we can find the corresponding values of $y_{t_{k}}$ and $v$. In addition, the values of $t_{k}$ and $k$ can also be determined, by using the regressive properties of $y_{n}$ and $y_{t_{n}}$. Let $k^{*}$ denote the largest value of $k$ such that $j\left(y_{t_{k}}, v\right) \in \rho_{x}$, for some number $v$. Then, it is easy to show that

$$
\begin{array}{ll}
w \in \varphi_{f}\left(\beta_{0}\right) \quad, \text { if } k^{*}=0, \text { and } \\
w \in \varphi_{f}\left(\beta_{k^{*}}\right)-\varphi_{f}\left(\beta_{k^{*}-1}\right), \text { if } k^{*} \geqq 1 .
\end{array}
$$

We know, by (25), that from the value of $y_{t_{k^{*}}}$ we can effectively find all the members of the set $\beta_{h^{*}}$. In addition, note that if $k^{*} \geqq 1$ then also the members of the set $\beta_{k^{*}-1}$ can be found, for we may regress down from $y_{t_{k^{*}}}$ to $y_{t_{k^{*}-1}}$ and apply (25). In a similar manner, in view of (14), it follows that from the value of $y_{t_{k^{*}}}$ we can find all the members in the set

$$
\begin{array}{ll}
\eta_{t_{0}} \quad, \text { if } k^{*}=0, \text { and } \\
\eta_{t_{k^{*}}}-\eta_{t_{k^{*}-1}}, & \text { if } k^{*} \geqq 1 .
\end{array}
$$

Finally, by combining these properties with the fact that the normal operators $\varphi_{f}$ and $\varphi_{g}$ are each recursive, it can be seen that the members in each of the sets below can be effectively determined,

$$
\begin{gathered}
\varphi_{f}\left(\beta_{0}\right) \text { and } \eta_{t_{0}}+\varphi_{g}\left(\beta_{0}\right), \quad \text { if } k^{*}=0 \text { and, } \\
\varphi_{f}\left(\beta_{k^{*}}\right)-\varphi_{f}\left(\beta_{k^{*}-1}\right) \quad \text { and } \\
\left(\eta_{t_{k^{*}}}-\eta_{t_{k^{*}-1}}\right)+\left(\varphi_{g}\left(\beta_{k^{*}}\right)-\varphi_{g}\left(\beta_{k^{*}-1}\right)\right), \quad \text { if } k^{*} \geqq 1 .
\end{gathered}
$$

It follows directly from this property and the definition of $q$, that the value of $q(w)$ can now be computed. Therefore, there will be a procedure that is effective and which will enable one to compute $q(w)$ for all but a possible finite number of $w \in \varphi_{f}(\beta)$. It is readily seen that this feature implies that the mapping $q$ will have a partial recursive extension. 
An approach very similar to the previous one can be employed to show that the mapping $q^{-1}$ will also have a partial recursive. For this reason we will omit the main details for doing this, and will only mention the two essentially new observatians that we would have been required to make. The first is that given any number $w \in \eta+\varphi_{g}(\beta)$ one can effectively determine whether $w \in \eta$ or $w \in \varphi_{g}(\beta)$. This property follows from the separability of the sets $\eta$ and $\varphi_{g}(\beta)$ given in (28). The other observation is that if $w \in \eta$, then one can effectively find the particular numbers $s, k^{*}, t_{k^{*}}$ and $y_{t_{k^{*}}}$ that are related to $w$ in the following way, $w=y_{s}$ and

$$
\begin{array}{ll}
w \in \eta_{t_{k^{*}}} & , \text { if } k^{*}=0, \\
w \in\left(\eta_{t_{k^{*}}}-\eta_{t_{k^{*}-1}}\right), \text { if } k^{*} \geqq 1 .
\end{array}
$$

This particular property follows from (14), (16), Property A and the regressive properties of the functions $y_{n}$ and $y_{t_{n}}$. The importance of the second property lies in the fact that it means that from the value of any $w \in \eta$, one can effectively find $y_{t_{k}}$, and therefore also determine the appropriate sets,

$$
\begin{array}{ll}
\beta_{t_{0}} \text { and } \eta_{t_{0}} & \text { if } k^{*}=0, \\
\beta_{t_{k^{*}}}, \beta_{t_{k^{*}-1}}, \eta_{t_{k^{*}}} \text { and } \eta_{t_{k^{*}-1}}, & \text { if } k^{*} \geqq 1 .
\end{array}
$$

It is then with these two observations that a similar approach, as with $q$, will lead to showing that $q^{-1}$ will have a partial recursive extension.

In view of the remarks made up to this point, we see that the mapping

$$
q: \varphi_{f}(\beta) \longrightarrow \eta+\varphi_{g}(\beta)
$$

will have a one-to-one partial recursive extension. This verifies (30) and complets the proof of the lemma.

Theorem 2. Let $f: E \rightarrow E$ be a recursive function and $A$ and $Y$ be isols such that

$$
D_{f}(A)=Y \text {. }
$$

If $Y$ is a regressive isol, then there will also exist regressive isols $B$ such that,

$$
D_{f}(B)=Y \text {. }
$$

Proof. Let us assume that $Y$ is a regressive isol. Let $f^{+}$and $f^{-}$be the positive and negative recursive and combinatorial functions that are associated with $f$ (refer to [11]). Then for every number $x \in E, f(x)=f^{+}(x)-f^{-}(x)$, and also 


$$
D_{f}(A)=D_{f}+(A)-D_{f}-(A) .
$$

Therefore, by (1), it also follows that

$$
D_{f}+(A)=Y+D_{f}-(A) .
$$

If we now apply the previous lemma to this equation, we see that there will be a regressive isol $B$ such that

$$
D_{f}+(B)=Y+D_{f}-(B),
$$

and from this identity it also follows that $D_{f}(B)=Y$.

REMARK. Theorem 2 is our principal result and it is easy to observe that it follows almost directly from the lemma. It turns out that, as a consequence of the manner in which the lemma was proved, a slightly stronger form of both the lemma and the theorem can be established. We would like to state without a proof the particular form that is related to the theorem. It involves the Nerode canonical extension of the familiar binary relation $\leqq$ (among numbers) to the isols. The extension procedure is introduced in [12], and for the relation $\leqq$ its extension will be denoted by $\leqq_{A^{*}}$. It can be shown that the regressive isol $B$ constructed in the proof of the lemma (in each of the cases considered there) is related to the isol $A$ by $B \leqq_{A}$ $A$. Based on this fact one can obtain the following result.

Theorem A. Let $f: E \rightarrow E$ be a recursive function and $A$ and $Y$ be isols such that $D_{f}(A)=Y$. If $Y$ is a regressive isol, then there will exist regressive isols $B$ such that $B \leqq{ }_{A} A$ and $D_{f}(B)=Y$.

3. An example. It is possible that the canonical extension of a recursive function may map an isol that is nonregressive onto an isol that is infinite and regressive. We would like to give an example of such a function. First some definitions are needed.

If $\alpha$ and $\beta$ are two sets of numbers, then $\alpha \leqq^{*} \beta$ will mean that either $\alpha$ is a finite set and card $\alpha \leqq \operatorname{card} \beta$, or else both $\alpha$ and $\beta$ are infinite sets and there is a partial recursive function $p$ such that, $\alpha \subseteq \delta p, p(\alpha)=\beta$ and $p$ is one-to-one on $\alpha$. If $A$ and $B$ are two isols then $A \leqq * B$ will mean that there are sets $\alpha \in A$ and $\beta \in \beta$ such that $\alpha \leqq * \beta$. Let $\min (a, b)$ denote the familiar recursive function minimum $(a, b)$, and let $D_{\min }$ denote its canonical extension to $\Lambda^{2} \cdot \min (a, b)$ is not an almost combinatorial function, and therefore its canonical extension will not map $\Lambda^{2}$ into $\Lambda$. On the otherhand, it is proved in [3] that $D_{\min }$ will map $\Lambda_{R}^{2}$ into $\Lambda_{R}$. In addition, by combining results in [3] and [4], one obtains for $A, B \in \Lambda_{R}$, 


$$
D_{\min }(A, B)=A \Longleftrightarrow A \leqq{ }^{*} B \text {. }
$$

Concerning isols and regressive isols the following property due to Dekker [4] is also needed; if $S$ and $T$ are any isols, then

$$
S \leqq T \text { and } T \in \Lambda_{R} \Longrightarrow S \in \Lambda_{R} .
$$

In the result below we will construct the kind of example that was described earlier. We note that the functions $j(x, y), k(x)$ and $l(x)$ that appear in its proof refer to those particular recursive functions introduced in $\S 1$.

THEOREM 3. There is a recursive function $h(x)$ and an isol $C$ such that $D_{h}(C) \in \Lambda_{R}$ and yet $C \notin \Lambda_{R}$.

Proof. Define

$$
h(x)=\min (k(x), l(x)) \text {. }
$$

Then $h$ will be a recursive function, and for $a, b \in E$

$$
h j(a, b)=\min (a, b) \text {. }
$$

Therefore also,

$$
D_{h} D_{j}(U, V)=D_{\min }(U, V), \text { for } U, V \in \Lambda .
$$

Select $A, B \in \Lambda_{R}$ such that

$$
A \leqq * B \text { and } A+B \notin \Lambda_{R} ;
$$

the existence of such a pair of regressive isols is proved in [2]. Then it follows

$$
D_{h} D_{j}(A, B)=D_{\min }(A, B)=A,
$$

and in addition, if we let $C=D_{j}(A, B)$, then also

$$
D_{h}(C)=A \in \Lambda_{R} \text {. }
$$

The function $j(x, y)$ is recursive and combinatorial, and therefore its canonical extension will map $\Lambda^{2}$ into $\Lambda$. In particular, we see that

$$
C=D_{j}(A, B) \in \Lambda \text {. }
$$

Let us now verify

$$
C=D_{j}(A, B) \in \Lambda_{R} \Longrightarrow A+B \in \Lambda_{R} .
$$

First consider the implications, 


$$
\begin{aligned}
D_{j}(A, B) \in \Lambda_{R} & \Longrightarrow 2 D_{j}(A, B) \in \Lambda_{R} \\
& \Longrightarrow 2 A+(A+B)(A+B+1) \in \Lambda_{R} \\
& =A+B \in \Lambda_{R} .
\end{aligned}
$$

The first two implications are clear. The last one follows from $(*)$ and the property,

$$
A+B \leqq 2 A+(A+B)(A+B+1) .
$$

Together they imply (4). In view of (1), (3) and (4) we obtain $C \in$ $\Lambda-\Lambda_{R}$, and if we combine this property with (2) the desired result follows directly.

$N$. $B$. The fact that the familiar $j$ function is combinatorial we first learned from some unpublished notes of Erik Ellentuck. Once this property is pointed out it is easy to show, and we will leave it for the reader.

4. Recursive functions of several variables. We would like to describe some of the results that can be obtained for recursive functions of more than one variable that are similar to those given in $\S 2$. First let us note some features that distinguish the one and more than one variable cases. We know that for a recursive combinatorial function of one variable, its canonical extension will map regressive isols onto regressive isols. On the otherhand, even for recursive combinatorial functions of two variables, it need not be true that their canonical extension will map pairs of regressive isols onto regressive isols. For example, Dekker showed in [4] that it is possible for both the sum and the product of two regressive isols to be an isol that is non-regressive. The characterization of those recursive functions of two variables whose canonical extensions will map regressive isols to regressive isols was given by Mathew Hassett in [9]. The following is a special case of a theorem also due to Hassett [8].

THEOREM B. (Hassett) Let $f: E^{n} \rightarrow E$ be a recursive and combinatorial function. Let $A_{1}, \cdots, A_{n}$ be $n$ regressive isols whose sum $A_{1}+\cdots+A_{n}$ is also regressive. Then the value of $D_{f}\left(A_{1}, \cdots, A_{n}\right)$ will be a regressive isol.

Note that when $n=1$ in Theorem $\mathrm{B}$ one obtains the earlier result mentioned about recursive combinatorial functions of one variable. Based upon the procedure for representing the canonical extension of a recursive function (in terms of the canonical extensions of recursive combinatorial functions) and applying Theorem $B$, analogues of Theorems 
1 and 2 can be obtained for functions of more than one variable. We conclude the paper with statements of these two theorems.

THEOREM C. Let $f: E^{n} \rightarrow E$ be a recursive function and $A_{1}, \cdots$, $A_{n}$ and $Y$ be isols with $D_{f}\left(A_{1}, \cdots, A_{n}\right)=Y$. If the sum $A_{1}+\cdots+$ $A_{n}$ is regressive, then the isol $Y$ will also be regressive.

THeOREM D. Let $f: E^{n} \rightarrow E$ be a recursive function and $A_{1}, \cdots$, $A_{n}$ and $Y$ be isols with $D_{f}\left(A_{1}, \cdots A_{n}\right)=Y$. If $Y$ is regressive, then there will be regressive isols $B_{1}, \cdots, B_{n}$ such that the sum $B_{1}+\cdots+B_{n}$ will be regressive and also $D_{f}\left(B_{1}, \cdots, B_{n}\right)=Y$.

\section{REFERENCES}

1. J. Barback, Recursive functions and regressive isols, Math. Scand., 15 (1964), 29-42.

2. - Two notes on regressive isols, Pacific J. Math., 16 (1966), 407-420.

3. Double series of isols, Canad. J. Math., 19 (1967), 1-15.

4. J. C. E. Dekker, The minimum of two regressive isols, Math. Zeit., 83 (1964), 345366 .

5. —, Regressive isols in Sets, Models and Recursion Theory, North-Holland, Amsterdam, 1967, pp. 272-296.

6. J. C. E. Dekker and J. Myhill, Recursive equivalence types, Univ. Calf. Publ. Math. (N. S)., 3 (1960), 67-213.

7. E. Ellentuck, Review of "Extensions to isols", by A. Nerode (see [12]), Math. Reviews, 24 (1962), \#A1215.

8. M. Hassett, A mapping property of regressive isols, Illinois J. Math., 14 (1970), 478487.

9. - A closure property of regressive isols, Rocky Mountain J. Math., 2 (1972), $1-24$.

10. J. Myhill, Recursive equivalence types and combinatorial functions, Bull. Amer. Math. Soc., 64 (1958), 373-376.

11. - Recursive equivalence types and combinatorial functions, Proc. 1960 Internat. Congress in Logic, Methodology and Philosophy of Science, Stanford Univ. Press, Stanford, Calf., (1962), 46-55.

12. A. Nerode, Extensions to isols, Ann. of Math., 75 (1962), 419-448.

13. F. J. Sansone, Combinatorial functions and regressive isols, Pacific J. Math., 13 (1963), 703-707.

Received May 21, 1971 and in revised form July 18, 1972. Research on the paper was supported in part by the National Science Foundation.

State University College of New York at Buffalo 


\section{PACIFIC JOURNAL OF MATHEMATICS}

\section{EDITORS}

\author{
H. SAMELSON \\ Stanford University \\ Stanford, California 94305 \\ C. R. HOBBY \\ University of Washington \\ Seattle, Washington 98105
}

\author{
J. DugundJI \\ Department of Mathematics \\ University of Southern California \\ Los Angeles, California 90007
}

\author{
RICHARD ARENS \\ University of California \\ Los Angeles, California 90024
}

\section{ASSOCIATE EDITORS}
E. F. BECKENBACH
B. H. NeumanN
F. WOLF
K. YoSHIDA

\section{SUPPORTING INSTITUTIONS}

\author{
UNIVERSITY OF BRITISH COLUMBIA \\ CALIFORNIA INSTITUTE OF TECHNOLOGY \\ UNIVERSITY OF CALIFORNIA \\ MONTANA STATE UNIVERSITY \\ UNIVERSITY OF NEVADA \\ NEW MEXICO STATE UNIVERSITY \\ OREGON STATE UNIVERSITY \\ UNIVERSITY OF OREGON \\ OSAKA UNIVERSITY
}

\author{
UNIVERSITY OF SOUTHERN CALIFORNIA \\ STANFORD UNIVERSITY \\ UNIVERSITY OF TOKYO \\ UNIVERSITY OF UTAH \\ WASHINGTON STATE UNIVERSITY \\ UNIVERSITY OF WASHINGTON \\ $* * * *$
$*$
AMERICAN MATHEMATICAL SOCIETY
NAVAL WEAPONS CENTER
}

The Supporting Institutions listed above contribute to the cost of publication of this Journal, but they are not owners or publishers and have no responsibility for its content or policies.

Mathematical papers intended for publication in the Pacific Journal of Mathematics should be in typed form or offset-reproduced, (not dittoed), double spaced with large margins. Underline Greek letters in red, German in green, and script in blue. The first paragraph or two must be capable of being used separately as a synopsis of the entire paper. The editorial "we" must not be used in the synopsis, and items of the bibliography should not be cited there unless absolutely necessary, in which case they must be identified by author and Journal, rather than by item number. Manuscripts, in duplicate if possible, may be sent to any one of the four editors. Please classify according to the scheme of Math. Rev. Index to Vol. 39. All other communications to the editors should be addressed to the managing editor, Richard Arens, University of California, Los Angeles, California, 90024.

50 reprints are provided free for each article; additional copies may be obtained at cost in multiples of 50 .

The Pacific Journal of Mathematics is published monthly. Effective with Volume 16 the price per volume (3 numbers) is $\$ 8.00$; single issues, $\$ 3.00$. Special price for current issues to individual faculty members of supporting institutions and to individual members of the American Mathematical Society: $\$ 4.00$ per volume; single issues $\$ 1.50$. Back numbers are available.

Subscriptions, orders for back numbers, and changes of address should be sent to Pacific Journal of Mathematics, 103 Highland Boulevard, Berkeley, California, 94708.

PUBLISHED BY PACIFIC JOURNAL OF MATHEMATICS, A NON-PROFIT CORPORATION

Printed at Kokusai Bunken Insatsusha (International Academic Printing Co., Ltd.), 270, 3-chome Totsuka-cho, Shinjuku-ku, Tokyo 160, Japan. 


\section{Pacific Journal of Mathematics}

\section{Vol. 43, No. 2 \\ April, 1972}

Arne P. Baartz and Gary Glenn Miller, Souslin's conjecture as a problem on the real line....................................... 277

Joseph Barback, On solutions in the regressive isols ............... 283

Barry H. Dayton, Homotopy and algebraic K-theory ................ 297

William Richard Derrick, Weighted convergence in length ............ 307

M. V. Deshpande and N. E. Joshi, Collectively compact and semi-compact sets of linear operators in topological vector spaces ............. 317

Samuel Ebenstein, Some $H^{p}$ spaces which are uncomplemented in $L^{p} \ldots . .327$

David Fremlin, On the completion of locally solid vector lattices ......... 341

Herbert Paul Halpern, Essential central spectrum and range for elements of

a von Neumann algebra............................... 349

G. D. Johnson, Superadditivity intervals and Boas' test ............. 381

Norman Lloyd Johnson, Derivation in infinite planes . . . . . . . . . . . 387

V. M. Klassen, The disappearing closed set property .............. 403

B. Kuttner and B. N. Sahney, On the absolute matrix summability of Fourier series ........................................... 407

George Maxwell, Algebras of normal matrices................... 421

Kelly Denis McKennon, Multipliers of type $(p, p) \ldots \ldots \ldots \ldots \ldots \ldots . . \ldots 29$

James Miller, Sequences of quasi-subordinate functions ............. 437

Leonhard Miller, The Hasse-Witt-matrix of special projective varieties ..... 443

Michael Cannon Mooney, A theorem on bounded analytic functions ...... 457

M. Ann Piech, Differential equations on abstract Wiener space .......... 465

Robert Piziak, Sesquilinear forms in infinite dimensions ............. 475

Muril Lynn Robertson, The equation $y^{\prime}(t)=F(t, y(g(t))) \ldots \ldots \ldots \ldots .483$

Leland Edward Rogers, Continua in which only semi-aposyndetic

subcontinua separate ............................... 493

Linda Preiss Rothschild, Bi-invariant pseudo-local operators on Lie

groups ...................................... 503

Raymond Earl Smithson and L. E. Ward, The fixed point property for

arcwise connected spaces: a correction ...................... 511

Linda Ruth Sons, Zeros of sums of series with Hadamard gaps .......... 515

Arne Stray, Interpolation sets for uniform algebras............... 525

Alessandro Figà-Talamanca and John Frederick Price, Applications of random Fourier series over compact groups to Fourier multipliers .. 